\title{
A rapid and simple bead-bashing-based method for genomic DNA extraction from mammalian tissue
}

\author{
Shan Wei', Brynn Levy²,3, Nataly Hoffman³ ${ }^{2}$ Claudia Cujar², Reunet Rodney-Sandy', Ronald Wapner', Mary D'Alton' \& Zev Williams ${ }^{*, 1}$
}

\section{ABSTRACT}

Conventional genomic DNA (gDNA) extraction methods can take hours to complete, may require fume hoods and represent the most time-consuming step in many gDNA-based molecular assays. We systematically optimized a bead bashingbased (BBB) approach for rapid gDNA extraction without the need for a fume hood. Human tissue specimens $(n=34)$ subjected to the 12-min BBB method yielded $0.40 \pm 0.17$ (mean $\pm \mathrm{SD}) \mu \mathrm{g}$ of gDNA per milligram of tissue, sufficient for many downstream applications, and 3- and 6-min extensions resulted in an additional $0.43 \pm 0.23 \mu \mathrm{g}$ and $0.48 \pm 0.43 \mu \mathrm{g}$ per milligram of tissue, respectively. The BBB method provides a simple and rapid method for gDNA extraction from mammalian tissue that is applicable to time-sensitive clinical applications.

\section{METHOD SUMMARY}

Extraction of genomic DNA is a critical and often rate-limiting step for many downstream genetic tests. However, existing methods can be time consuming, difficult to automate and require use of a fume hood. Here, we present a simple 12-min method for genomic DNA extraction from tissue without the need for a fume hood and which can be readily automated for high-throughput applications.

\section{KEYWORDS}

genetic testing $\cdot$ rapid clinical diagnostics - rapid tissue gDNA extraction - tissue processing

${ }^{1}$ Department of Obstetrics \& Gynecology, Columbia University Irving Medical Center, New York, NY 10032, USA; ${ }^{2}$ Department of Pathology \& Cell Biology, Columbia University Medical Center, New York, NY 10032, USA; ${ }^{3}$ Clinical Cytogenetics Laboratory, Columbia University Medical Center \& the New York Presbyterian Hospital, New York, NY 10032,USA; *Author for correspondence:zw2421@ cumc.columbia.edu

BioTechniques 68: 240-244 (May 2020) 10.2144/ btn-2019-0172
Extracting high-quality genomic DNA (gDNA) from tissue is the first step for many molecular genetic studies and clinical diagnostic tests [1-5]. For rapid clinical genetic tests, such as qPCR, PCR-based genotyping and nanopore-based sequencing, the DNA extraction step is now the most time-consuming component of the workflow and adds significant time to the testing [1,6-11]. Extraction of gDNA from mammalian tissue typically uses detergents and/or enzymes to lyse the cell and nuclear membranes, providing DNA yields of approximately $0.2-3 \mu \mathrm{g}$ of gDNA per milligram of tissue, depending on the tissue [12]. However, the lysis step requires between 2 and $12 \mathrm{~h}$ to complete. Because incomplete lysis will lead to a decrease in DNA yield and an overnight lysis leads to higher gDNA yields without decreasing DNA quality $[13,14]$, the standard protocol for many basic and clinical labs is to use overnight lysis to ensure the quality and yield of gDNA extracted from mammalian tissues $[5,13,15]$. Liquid nitrogen and tissue disruption using a syringe have also been used to enhance the homogenization of human tissue samples and shorten lysis processing times to approximately $1 \mathrm{~h}$ $[16,17]$. However, these methods require more hands-on time, have a low throughput and are not amenable to automation (Figure 1). While traditional bead-bashing methods for DNA extraction have been applied to samples that are difficult to extract, such as insect cells [18], they have required the use of $\beta$-mercaptoethanol ( $\beta-\mathrm{ME})$, a fume hood and a more complex multi-step procedure using multiple DNA purification columns, which is impractical for use with automation and/or in a clinical setting (Figure 1).

In this study, we report a simplified bead-bashing-based (BBB) method for rapidly extracting high-quality gDNA from human tissue. This simplified workflow streamlines the gDNA extraction process and provides sufficient high-quality gDNA for in under $15 \mathrm{~min}$. An enhanced workflow also provides more than triple the gDNA yield to accommodate applications requiring higher input DNA quantities.

\section{MATERIALS \& METHODS}

Genomic DNA was extracted from approximately $2 \mathrm{mg}$ samples of chorionic villi. Tissues were weighed in an analytical scale (Mettler, AE 100), placed in ZR BashingBead Lysis Tubes (2.0 mm) (Zymo, D6015), and mixed with $550 \mu$ l BashingBead Buffer (Zymo, D6015) and $10 \mu$ l RNase A 20 mg/ml (Invitrogen, 12091021). Each sample was vortexed on a vortex mixer (Fisher, 02-215-422) at maximum speed (3000 rpm) for $7 \mathrm{~min}$. Should faster treatment be needed, a high-speed cell disrupter such as FastPrep-24 (maximum speed $4750 \mathrm{rpm}$ ) or BeadRuptor 24 (maximum speed 5000 rpm) can be used to complete the homogenizing process in 3-5 min, as suggested by the manufacturer's protocol (Zymo, D6015), with optimization based on tissue type. Each homogenized sample was subjected to centrifugation at 12,000 rcf for $1 \mathrm{~min}$ (Eppendorf centrifuge 5424), and $200 \mu \mathrm{l}$ supernatant was retrieved for column purification. This step allows the retrieval of approximately $0.4 \mu \mathrm{g}$ DNA per mg tissue (Figure 1).

Should additional gDNA be needed for downstream analysis, a 3-min extension may be used with an additional $200 \mu \mathrm{l}$ bashing bead buffer added to each original ZR BashingBead lysis tube, vortexed for $30 \mathrm{~s}$ and the supernatant was recovered as above. Should still more gDNA be needed, another 6-min extension may be used by repeating the additional $200 \mu \mathrm{l}$ bashing bead buffer bead-bashing step. Total gDNA yields per sample represent the cumulative gDNA recovered from these elutions (Supplementary Figure 1). 


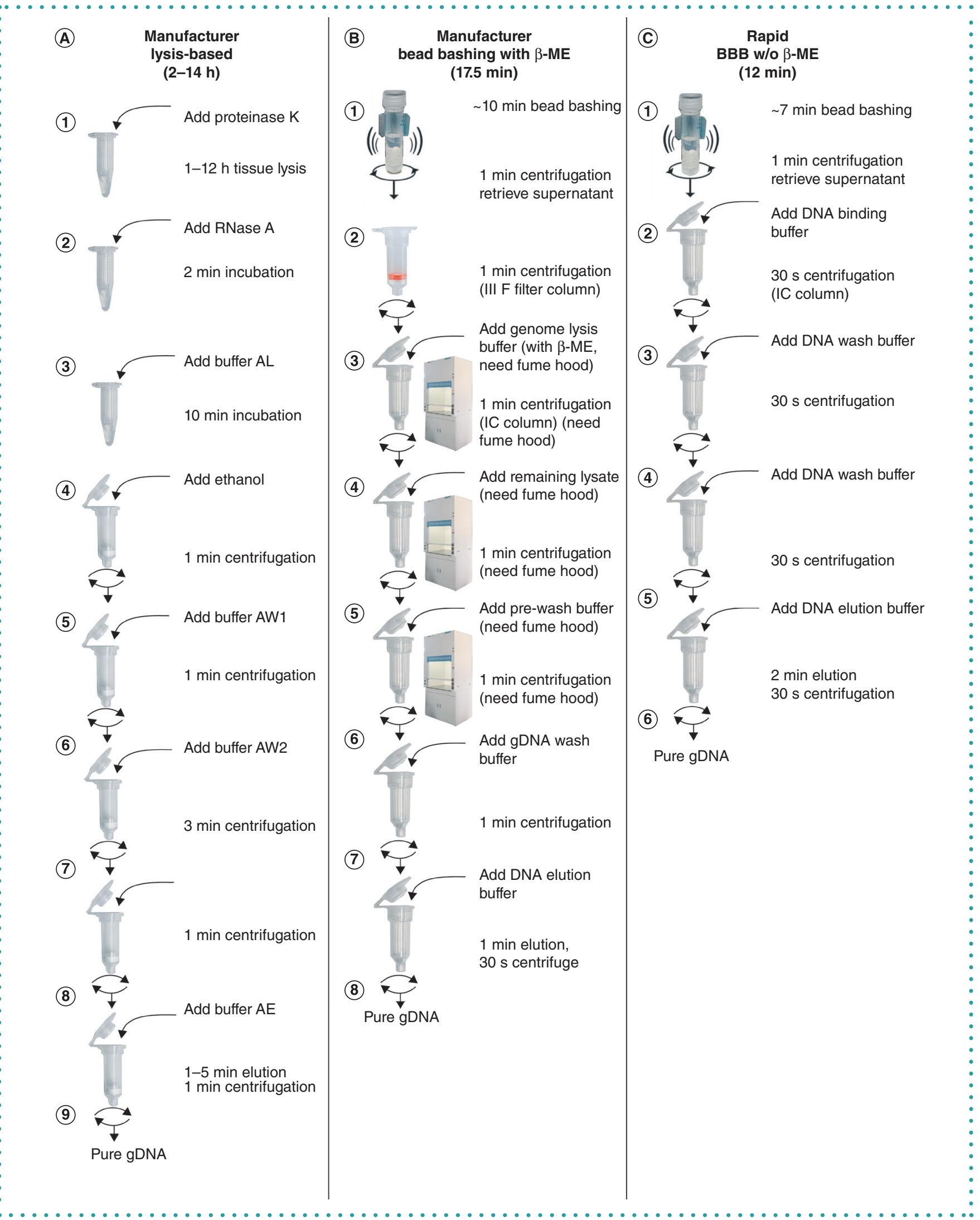

Figure 1. Comparison of workflows between the manufacturer's lysis-based and bead bashing-based, and the rapid bead bashing-based protocol. Images of each module were adapted from manufacturer's protocols. (A) Workflow illustration remade from manufacturer's protocol in QIAamp DNA mini kit for tissue. (B) Workflow illustration remade from manufacturer's protocol in Quick-DNA Insect/Tissue kit. (C) Workflow illustration of rapid bead-bashing-based gDNA extraction methods reported in this study. 


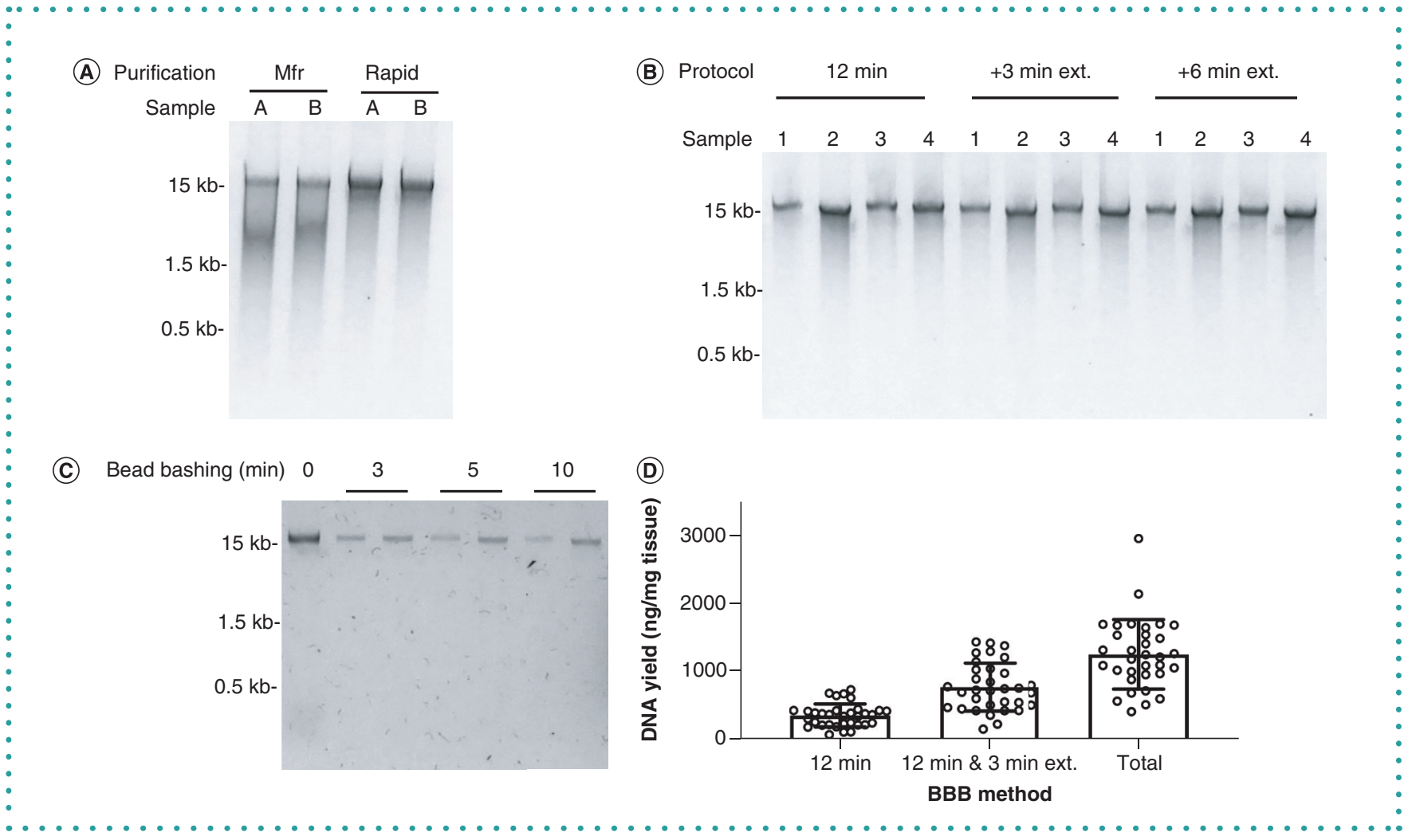

Figure 2. Development of rapid bead bashing-based method and extensions. (A) Comparison of gDNA purified using two $\beta$-mercaptoethanol-free protocols by gel electrophoresis. Two tissue samples (A \& B) were purified using the Quick-DNA insect/tissue kit protocol (Mfr) and DNA Clean \& Concentrator-5 protocol (rapid). (B) Gel image of gDNA extracted from chronic villus tissue samples using the rapid and extended BBB protocols. Tissue samples (samples 1-4) were subjected to the rapid BBB method as well as a 3-and 6-min extension. (C) Evaluation of the impact of the bead-bashing process on the integrity of gDNA. Technical repeats of gDNA were treated for 3,5 and $10 \mathrm{~min}$ bead bashing times. (D) gDNA yields using rapid and extended BBB methods. gDNA yields from each individual sample are shown by dots; the bar graph indicates the mean value \pm SD. BBB: Bead bashing-based.

To perform column purification, supernatants were mixed with threefold volume of DNA Binding Buffer (Zymo, D4013) at room temperature $\left(25^{\circ} \mathrm{C}\right)$, and then loaded on Zymo IC Spin columns (assembled in a clean $2 \mathrm{ml}$ collection tube) (Zymo, D4013). Spin columns were centrifuged at 10,000 rcf for $30 \mathrm{~s}$, then washed in first $500 \mu \mathrm{l}$ and then $200 \mu \mathrm{l}$ DNA Wash Buffer (Zymo, D4013) at $10,000 \mathrm{rcf}$ for $30 \mathrm{~s}$ each. The columns containing ultrapure gDNA were then incubated with $40 \mu \mathrm{l}$ Zymo DNA elution buffer (10 mM Tris-HCl, pH 8.5, 0.1 mM EDTA) (Zymo, D4013) at $37^{\circ} \mathrm{C}$ for $1-3 \mathrm{~min}$, and then centrifuged at 10,000 rcf for $30 \mathrm{~s}$ in a clean low-retention microcentrifuge tube $(1.5 \mathrm{ml})$.

The quality of gDNA was examined on a $0.8 \%$ agarose gel, and the concentration was determined by a Qubit dsDNA HS assay kit (Invitrogen, Q32851). A comparative study with the Zymo Quick-DNA ${ }^{\text {tw }}$ Tissue/Insect kit (Zymo, D6015) was performed according to the manufacturer's protocol. The study was approved by the Columbia University Institutional Review Board.

\section{RESULTS \& DISCUSSION}

The Quick-DNA ${ }^{\text {mm }}$ Tissue/Insect Microprep Kit (Zymo, D6015) requires the use of $\beta-M E$, a fume hood, multiple columns and purification steps that increase hands-on time and make automation - important for clinical application - challenging (Figure 1). We first tried omitting $\beta$-ME; however, this resulted in poor DNA quality (Figure 2A). To optimize the purification of gDNA following bead bashing without the need for $\beta$-ME, we next compared the manufacturer's purification protocol with a direct DNA Clean \& Concentrator-5 (Zymo, D4013) protocol. Compared with the manufacturer's protocol, the direct DNA Clean \& Concentrator-5 protocol has fewer centrifugation steps (5 vs 7), a shorter centrifugation time (30 s vs $1 \mathrm{~min}$ ), and overall requires less time than the kit's protocol (12 vs $17.5 \mathrm{~min}$ ) (Figure 1
\& Supplementary Figure 1). It also only uses a single column (IC column vs III F filter + IC column) and one wash buffer (DNA Wash Buffer vs DNA Pre-Wash Buffer + gDNA Wash Buffer) (Figure 1). The direct DNA Clean \& Concentrator-5 protocol recovered a similar amount of gDNA as the original kit, but yielded larger fragments $(>15 \mathrm{~kb})$ of intact gDNA instead of smeared DNA (Figure 2A). Hence, the direct DNA Clean \& Concentrator-5 is more suitable for purification of gDNA from human soft tissue in combination with a 7-8 min bead-bashing treatment. The Quick-DNA Tissue/insect kit uses the selective chemistry of different washing buffers to eliminate RNA, but this protocol is insufficient to remove impurities in gDNA from human soft tissues without the addition of $\beta$-ME (Figure 2A). To eliminate these steps, we added RNase $A$ during the bead-bashing step so that a simple DNA Clean \& Concentrator-5 protocol with one DNA wash buffer is sufficient to yield clean 
intact gDNA (Figure 1 \& Figure 2A).

We next tested the 12-min rapid, and 3and 6-min extended BBB gDNA protocols on 34 chorionic villus tissue specimens (Supplementary Table 1). gDNA quality was examined by gel electrophoresis (Figure $2 \mathrm{~B}$ ). Using the 12-min rapid BBB protocol, $0.40 \pm 0.17 \mu \mathrm{g}$ (mean \pm standard deviation) of gDNA were extracted per milligram of tissue (range: $0.11-0.77 \mu \mathrm{g}$ per $\mathrm{mg}$ of tissue), sufficient for downstream applications such as qPCR, MLPA, microarray analysis and nanoporebased sequencing, and similar to gDNA yields using the manufacturer's protocol (1-3 $\mu$ g gDNA per mg tissue) (Figure 2D and Supplementary Table 1). The 3- and 6-min extended protocols resulted in an additional $0.43 \pm 0.23 \mu \mathrm{g}$ (range: $0.08-1.05 \mu \mathrm{g}$ ) and $0.48 \pm 0.43 \mu \mathrm{g}$ (range: $0.16-2.45 \mu \mathrm{g}$ ) per milligram of tissue, respectively, sufficient yields for whole genome sequencing, genotyping and other downstream analysis (Figure 2D \& Supplementary Table 1). In total, 0.4-3 $\mu$ g gDNA were extracted per milligram of tissue samples using rapid BBB methods with the 6-min extensions, comparable with commercial kits such as QIAamp DNA Mini Kit (Qiagen, 51304: 0.2-3 $\mu$ g per mg of tissue) and PureLink gDNA kit (Invitrogen, K182001: 0.4-4 $\mu$ g per mg tissue).

Two nonsystemic factors can contribute to the large standard deviation of gDNA yields reported in this study. The first is the low DNA input. The suggested input of this method is $\mathbf{2}-\mathbf{5} \mathrm{mg}$ tissue, making it more sensitive to medium/buffer retention than a $20 \mathrm{mg}$ tissue sample. Retention of $1 \mu \mathrm{l}$ liquid will lead to an approximately $1 \mathrm{mg}$ increase in estimated weight. Second, there was variation inherent in the samples. The method was performed on actual clinical samples and there may have been variability in the initial processing and handling of these samples that could impact their DNA yield. However, the gDNA yields across all samples were within the range of expected yield for the commercial kits, and sufficient for gDNA-based assay. In this study, sample 26 produced the lowest gDNA yield using the rapid and extended BBB methods. That could have been caused by an overestimation of the sample weight, or low amounts of genetic material in the initial specimen. However, even in this sample the gDNA yield was sufficient for nanopore sequencing or PCR-based assays.
Finally, we investigated the effect of the bead-bashing time on the integrity of large gDNA fragments in lieu of multi-hour lysis steps. gDNA (100 ng) in TE buffer was subjected to bead-bashing at $3000 \mathrm{rpm}$ for 3,5 and $10 \mathrm{~min}$; examination of a $0.8 \%$ agarose gel confirmed that 10 min of the bead-bashing process did not result in an increased amount of sheared DNA (Figure 2C).

The rapid $B B B$ methods showed promising results for gDNA extraction from human soft tissue in under $20 \mathrm{~min}$. The 12-min rapid BBB method yields sufficiently large fragment size (>15 kb) gDNA for applications that require $100 \mathrm{ng}$ gDNA or less $[1,6,11]$. The 3 - and 6 -min extensions recovered more gDNA from the same specimen to accommodate applications that requires sub- $\mu \mathrm{g}$ to $\mu \mathrm{g}$-level gDNA input $[5,19]$. The rapid BBB gDNA extraction method significantly reduces the time, complexity and equipment required to extract high-quality gDNA from tissue, and allows urgent gDNAbased diagnostic tests to be carried out in a timely manner. However, the BBB methods will need further validation and testing of different tissues.

The rapid BBB method can provide largesize gDNA from clinical human specimens in under $15 \mathrm{~min}$, which can assist rapid gDNAbased clinical diagnostic assays and significantly shorten the workflow. The addition of a 3-or 6-min extension to the protocol can also provide sufficient gDNA for applications requiring higher input. Overall, this is a rapid yet efficient and versatile tissue gDNA extraction method for clinical and basic research.

\section{FUTURE PERSPECTIVE}

The clinical applications of rapid DNA-based diagnostic techniques such as qPCR, PCR-based genotyping, and nanopore sequencing have surged, and with them an increasing need for faster methods for extracting genomic DNA (gDNA) from tissue. The protracted time required for DNA extraction delays the turnaround time for many molecular assays, including urgent and time-sensitive applications where return of results in the same day or even within hours is of important clinical value. A simple, robust and rapid gDNA extraction method can significantly speed up rapid clinical diagnostics and make same-day results accessible.

\section{AUTHOR CONTRIBUTIONS}

$S$ Wei developed the method, performed experiments, managed the project and prepared the manuscripts. B Levy participated in experiment design, project coordination, sample recruitment and manuscript preparation. $\mathrm{N}$ Hoffman and $\mathrm{C}$ Cujar participated in sample collection and manuscript preparation. R Rodney-Sandy participated in experiments and manuscript preparation. $\mathrm{R}$ Wapner and M D'Alton participated in experiment design and manuscript preparation. Z Williams conceived and oversaw the project, and participated in method development, project management and manuscript preparation.

\section{SUPPLEMENTARY DATA}

To view the supplementary data that accompany this paper please visit the journal website at: www.future-science. com/doi/suppl/10.2144/btn-2019-0172

\section{ACKNOWLEDGMENTS}

The authors thank members of the Williams' Laboratory, Columbia University Fertility Center, and the Clinical Cytogenetics Laboratory at Columbia University Medical Center \& the New York Presbyterian Hospital for their helpful input and advice with this project and manuscript.

\section{FINANCIAL \& COMPETING INTERESTS DISCLOSURE}

$S$ Wei and Z Williams are listed as inventors on patents relating to this technology filed by Columbia University. This research was supported by the National Institutes of Health grants HD068546, HD100013 and U19CA179564, and Columbia BioMedX. The authors have no other relevant affiliations or financial involvement with any organization or entity with a financial interest in or financial conflict with the subject matter or materials discussed in the manuscript apart from those disclosed.

No writing assistance was utilized in the production of this manuscript.

ETHICAL CONDUCT OF RESEARCH The study was approved by the Columbia University Institutional Review Board. 


\section{OPEN ACCESS}

This work is licensed under the AttributionNonCommercial-NoDerivatives 4.0 Unported License. To view a copy of this license, visit http://creativecommons.org/licenses/ by-nc-nd/4.0/

\section{REFERENCES}

Papers of special note have been highlighted as: - of interest

1. Zhang J, Walsh MF, Wu G et al. Germline mutations in predisposition genes in pediatric cancer. N. Engl. J. Med. predisposition genes in pediatric

- A simple, rapid and robust gDNA extraction method from mammalian tissue can be applied on and can significantly speed up the the listed studies.

2. Carp H, Guetta E, Dorf H, Soriano D, Barkai G, Schiff E. Embryonic karyotype in recurrent miscarriage with parental karyotypic aberrations. Fertil. Steril. 85(2), 446-450 (2006).

3. Palomaki GE, Deciu C, Kloza EM et al. DNA sequencing of maternal plasma reliably identifies trisomy 18 and trisomy 13 as well as Down syndrome: an international trisomy 13 as well as Down syndrome: an international
collaborative study. Genet. Med. 14(3), 296-305 (2012).

4. De Paoli-Iseppi R, Johansson PA, Menzies AM et al. Comparison of whole-exome sequencing of matched fresh and formalin fixed paraffin embedded melanoma tumours: implications for clinical decision making. Pathology 48(3), 261-266 (2016).
5. Levy B, Wapner R. Prenatal diagnosis by chromosomal microarray analysis. Fertil. Steril. 109(2), 201-212 (2018).

- A simple, rapid and robust gDNA extraction method from mammalian tissue can be applied on and can significantly speed up the the listed studies.

6. Wei $\mathrm{S}$, Weiss ZR, Gaur P, Forman E, Williams Z. Rapid preimplantation genetic screening using a handheld preimplantation genetic screening using a handheld,
nanopore-based DNA sequencer. Fertil. Steril. 110(5), nanopore-based DNA
910-916 e912 (2018)

7. Minervini $C F$, Cumbo $C$, Orsini $P$ et al. TP53 gene mutation analysis in chronic lymphocytic leukemia by nanopore MinION sequencing. Diagn. Pathol. 11(1), 96 (2016).

8. Quick J, Grubaugh ND, Pullan ST et al. Multiplex PCR method for MinION and Illumina sequencing of Zika and other virus genomes directly from clinical samples. Nat. Protoc. 12(6), 1261-1276 (2017).

9. Miller FJ, Rosenfeldt FL, Zhang C, Linnane AW, Nagley P. Precise determination of mitochondrial DNA copy number in human skeletal and cardiac muscle by a PCR based assay: lack of change of copy number with age. Nucleic Acids Res. 31(11), e61 (2003).

- A simple, rapid and robust gDNA extraction method from mammalian tissue can be applied on and can significantly speed up the the listed studies.

10. Wei S, Williams Z. Rapid short-read sequencing and aneuploidy detection using MinION nanopore technology. Genetics 202(1), 37-44 (2016).

11. Wei S, Weiss ZR, Williams Z. Rapid multiplex small DNA sequencing on the MinION nanopore sequencing platform. G3 (Bethesda) 8(5), 1649-1657 (2018).

- A simple, rapid and robust gDNA extraction method from mammalian tissue can be applied on and can significantly speed up the the listed studies.
12. Tomaso H, Kattar M, Eickhoff $\mathrm{M}$ et al. Comparison of commercial DNA preparation kits for the detection of Brucellae in tissue using quantitative real-time PCR. BMC Infect. Dis. 10, 100 (2010).

13. Roy-Chowdhuri S, Chow CW, Kane MK et al. Optimizing the DNA yield for molecular analysis from cytologic preparations. Cancer Cytopathol. 124(4), 254-260 (2016).

14. Majumdar G, Vera S, Elam MB, Raghow R. A streamlined protocol for extracting RNA and genomic DNA from archived human blood and muscle. Anal. Biochem. 474, 25-27 (2015).

15. Pearson $\mathrm{H}$, Stirling D. DNA extraction from tissue. In: PCR Protocols. Bartlett JMS, Stirling D (Eds). Humana Press, NJ, USA, 33-34 (2003).

16. Pajni $\bigotimes$ IZ. Extraction of DNA from human skeletal material. In: Forensic DNA Typing Protocols. Goodwin W (Ed.). 89-108 Springer, NY, USA (2016).

17. Wu Q, Chen $M$, Buchwald M, Phillips RA. A simple, rapid method for isolation of high quality genomic DNA from animal tissues. Nucleic Acids Res. 23(24), 5087-5088 (1995).

- A simple, rapid and robust gDNA extraction method from mammalian tissue can be applied on and can significantly speed up the the listed studies.

18. Marr EJ, Wright HW, Sargison ND, Nisbet AJ, Burgess STG. Gene silencing by RNA interference in the ectoparasitic mite, Psoroptes ovis. Vet. Res. 49(1), 112 (2018).

19. Wapner RJ, Martin CL, Levy B et al. Chromosomal microarray versus karyotyping for prenatal diagnosis. $N$ Engl. J. Med. 367(23), 2175-2184 (2012).

- A simple, rapid and robust gDNA extraction method from mammalian tissue can be applied on and can significantly speed up the the listed studies. 